\title{
Cardiac output at skin level: PPC vs. Fick method
}

\begin{abstract}
Hemodynamic monitoring is essential in the therapeutic guide of the critical patient. The pulsomagneto cardiac pressure gauge (PPC), is a non-invasive device, which records the cardiac output $(\mathrm{CO})$ at the skin level and continuously. The objective of this work is determining the correlation of the GC measurement between the PPC system and the Fick Method in patients in the intensive care unit (ICU). It is a prospective, observational and longitudinal study. The $\mathrm{CO}$ was measured with both methods in 121 patients at admission, in addition to performing an analysis with Bland-Altman. As a Result of this work, the means of the $\mathrm{CO}$ by both methods did not show statistical differences $\mathrm{p}=0.05$. The Sperman test reported a coefficient $\mathrm{r}=0.025$, not significant $\mathrm{p}=0.77$. The Bland-Altman analysis shows that the PPC overestimates the value of the $\mathrm{GC}$ at $3 \mathrm{~L}$. The results suggest that an imperfect standard was chosen. The pulse-magneto cardiac pressure gauge is operator-dependent. The $\mathrm{PPC}$ is a portable device for non-invasive monitoring of GC in critical patients.
\end{abstract}

Keywords: cardiac output (GC), pulsomagneto cardiac pressure gauge (PPC), Fick's method
Volume 6 Issue 5 - 2020

\author{
Alba Hernández-Gutierrez,,2 Martha Alicia \\ Hernández-González, ${ }^{3}$ Teodoro Córdova- \\ Fraga, ${ }^{4}$ Pedro Luis González-Carrillo, ${ }^{2}$ \\ Angélica Hernández-Rayas, ${ }^{4}$ Rafael Guzman- \\ Cabrera ${ }^{5}$ \\ 'Unidad de Terapia Intensiva, México \\ ${ }^{2}$ División Ciencias de la Salud, Universidad de Guanajuato, \\ México \\ ${ }^{3}$ División en Investigación en Salud, Unidad Médica de Alta \\ Especialidad, México \\ ${ }^{4}$ Departamento de Ingeniería Física - DCl, Universidad de \\ Guanajuato campus León, México \\ ${ }^{5}$ Departamento de Ingeniería Eléctrica - DICIS, Universidad de \\ Guanajuato, México
}

\begin{abstract}
Correspondence: Rafael Guzman-Cabrera, Departamento de Ingeniería Eléctrica - DICIS, Universidad de Guanajuato, Carretera Salamanca -Valle de Santiago km 3.5 + I.8 Comunidad de Palo Blanco, 36885, Salamanca, GTO, México Email guzmanc@ugto.mx
\end{abstract}

Received: November 23, 2020 | Published: December 2I, Receiver
2020

\section{Introduction}

The evaluation of the hemodynamic state of the patient in critical condition is essential to provide the best treatment alternative and establish the prognosis of the disease, particularly in those hospitalized in the areas of Intensive Care, where the overall mortality ranges from 28 to $30 \% .^{1}$ The main objective of hemodynamic management is based on tissue perfusion, which is achieved by optimizing the recording of cardiac output with its determinants and arterial oxygen content. In such a way that the Hospital must have the tools to monitor the parameters that allow on the one hand to establish precise diagnoses, such as hypovolemia, heart failure, vasoplegia, cardiac or pericardial tamponade, etc .; and on the other hand to guide the treatment established (administration of fluids, use of inotropics, vasopressors). These variables should be relevant to the clinical situation, with interpretable data, easy to use and without risks. Theoretical knowledge and experience has allowed the assessment of the hemodynamic state of various clinical situations, through a practical management based on simple variables, ${ }^{2,3}$ such as serial measurement of blood pressure, central venous pressure, electrocardiographic monitoring and fundamentally the clinical assessment (awareness, skin characteristics, heart rate, peripheral perfusion, urinary expenditure, etc.) Although frequently, these parameters are insufficient for adequate therapeutic management, being altered only when the compensatory mechanisms have failed in their mission of preserving the integrity of the organism.

The technology that allows hemodynamic monitoring is constantly evolving and changing, due to the advancement in computer science, nanotechnology and the advent of portable devices that allow expanding the range of tools available in the patient's bedside. Thus, we pass from an invasive global hemodynamic monitoring to a non-invasive and local-regional one, including tissue, ${ }^{4}$ In parallel, the combined evolution of technology and physiological and pathophysiological concepts offer clinicians access to functional hemodynamic monitoring that allows the analysis of dynamic variables, providing greater accuracy in information, especially the state of vollemia, allowing to predict The need to administer fluids.

Invasive monitoring techniques such as the Swan-Ganz catheter and the central venous catheter are bloody, not without complications and in some cases, of questioned benefit, so the doctor has been forced to have to take an additional risk every Once you've decided to use them. Other methods that help to understand the hemodynamic behavior of vascular flow are Doppler echocardiography and cardiovascular magnetic resonance, however, they are methods that are not used routinely and are unable to record the behavior of vascular flow continuously. ${ }^{5}$ For this reason, in recent years, in the light of new discoveries in the understanding of pathophysiological processes, there has been a growing interest in the development of new devices that provide an effective alternative to classical monitoring methods by applying Non-invasive or minimally invasive techniques that demonstrate results comparable to the diagnostic reference procedures such as the Magneto-Cardiac Pressure Gauge (PPC).

\section{Biomagnetism and measurement of vascular pressure}

In the Division of Sciences and Engineering of the University of Guanajuato, a portable device has been developed to measure cardiac output by means of electromagnetism in a non-invasive way and at skin level, ${ }^{6,7}$ where the resistive magneto effect is present, which is the property by which, a ferromagnetic material changes its resistance $\mathrm{R}$, in the presence of an external magnetic field. The resistance $\mathrm{R}$ of the permaloy changes depending on the angle of rotation, given by:

$$
R=R_{0}+\Delta R_{0} \cos ^{2} \alpha
$$


where $\mathrm{R} 0$ and $\Delta \mathrm{R} 0$ are the characteristic parameters of the sensor material. ${ }^{8}$

This allows continuous measurement of linear and angular displacements with precision of $\mathrm{nT}$, that is, small mechanical movements of the magnet in the order of tenths of a millimeter, such as those that occur in the arterial and venous wall due to changes in intravascular pressure and due to the distensibility of the vessel they produce measurable changes in the magnetic field. ${ }^{9}$ It has already been shown that there is an adequate correlation of the measurement of systemic blood pressure between the sphygmomanometer with the biomagnetism by pulse-magneto cardiac pressure gauge (PPC) in healthy volunteers. ${ }^{10,11}$ On the other hand, considering that the recording of cardiac output is important, this in severe patients, it is expected that this device works to identify their hemodynamic state in a continuous and non-invasive way, as well as to be able to provide them with the best treatment alternative. It is necessary to know if the measurement of cardiac output by biomagnetism is similar to that obtained with invasive devices (Fick's method by means of arterial and venous gasometries, as well as the different parameters recorded by PVC by means of a central venous catheter) and not Conventional invasive (sphygmomanometer). In particular, the PPC is a portable, non-invasive device, which in healthy volunteers is able to record the amplitude of the venous and arterial pulse continuously. The reliability of the PPC to measure cardiac output, taking as a reference the techniques considered as gold standard in severe patients is presented, the measurement protocol is also proposed so that this device could be a useful diagnostic alternative in Intensive Care Units, with all the advantages of continuous and non-invasive monitoring.

\section{Materials and methods}

A prospective, longitudinal and analytical study of the correlation of diagnostic test was performed in adult patients admitted to the intensive care unit, whose clinical conditions warrant hemodynamic monitoring of cardiac output through a gas workshop, once it was authorized by the ethics committee and research of the Hospital. Patients older than 18 years were included, who accepted, or failing that, a family member or guardian, to participate in the study by signing the informed consent, hospitalized in the intensive care unit of the National Medical Center of Bajío UMAE 1. Pregnant patients were excluded, patients with tracheostomy, with a diagnosis of congenital heart disease, neck injuries (burns, wounds or sites of infection), who did not have a central venous catheter or anatomical site not accessible for taking arterial puncture. Cardiac output following Fick's method and with the PPC was measured simultaneously and within the first 4 hours of admission to the ICU. To obtain the cardiac output by the PPC: a cylindrical magnet $3 \mathrm{~mm}$ in diameter and $4 \mathrm{~mm}$ high, with a magnetic moment of $0.013 \mathrm{~A} / \mathrm{m} 2$, was attached to the patient's skin, so the records are kept level of skin, just above the left or right carotid, where the pulse is adequately palpated, and through a magneto resistor sensor the variation of the movement of the magnet caused by the transmission of the pulse is recorded. This signal is sent to a module, which by means of a micro controller amplifies and converts the signal from analog to digital. Finally, it is recorded on a laptop to visualize the curve by means of software programmed to acquire and save the data of the cardiac output measurement, whose values are expressed in liters per minute (Figure 1).

To obtain cardiac output by Fick's method, two gasometries, arterial and central venous, will be obtained, applying the GC formula: $\mathrm{VO} 2(\mathrm{CaO}-\mathrm{CvO} 2)$. Both measurements of different techniques were performed simultaneously. The results obtained were emptied into a capture sheet in Excel format and using the statistical packages SPSS v20 and Med Calc v19, the comparison of both methods was performed using the Student $t$ test for dependent samples and the Pearson Correlation coefficient. For objectivity of the utility of the PPC, an analysis was carried out under the ROC curve using the Hanley test, considering the Fick method as a reference test and finally the concordance of both procedures with the Bland Altman test. For all inferential statistical analyzes, values of $p<0.05$ were considered significant. The utility of the test was calculated by analyzing the ROC curve by the Hanley test.

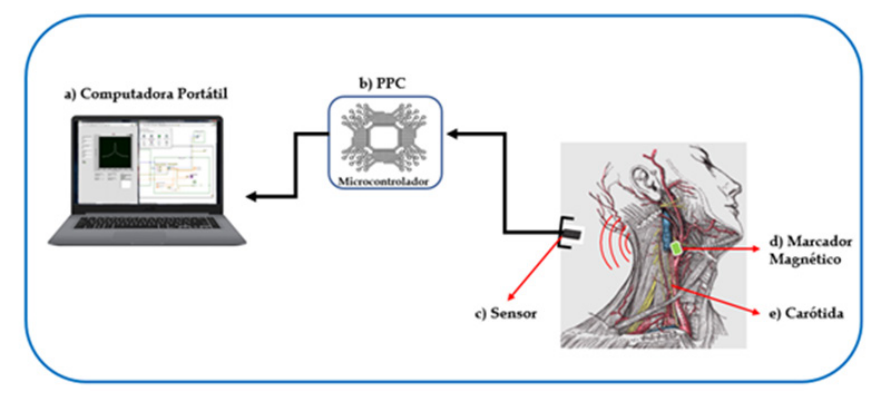

Figure I Scheme for monitoring cardiac output with PPC.A) Laptop, B) PPC consisting of a microcontroller and circuitry, C) Magnetic Sensor, D) Magnetic marker and E) Carotid Location.

\section{Results}

Therecords weremade for elevenmonths, 121 patients were included (Figure 2), with a mean age of 44.6 years. With a higher prevalence of the male gender, in a total of 70 men corresponding to $57.85 \%$, the average of APACHE II and SOFA were 19.9 and 10.15 points, respectively; the diagnosis of admission with the highest incidence was Subarachnoid Hemorrhage 29 patients (35\%), the percentage distribution of the incidence of the diagnoses is described in Figure 3. The comparison of the average cardiac output obtained by the PPC was compared with the one that the Fick method showed (10.42 \pm 13.41 vs. $10.17 \pm 10.55$ respectively), without finding significant statistical differences $(p=0.06)$ (Figure 4$)$. When the correlation between the two procedures was made using the Spearman test, a coefficient $r=$ 0.025 (95\% CI -0.1534-0.2033) was found, not significant $(\mathrm{p}=0.77)$. (Figure 5) The concordance between the two methods was evaluated by Bland-Altman analysis, finding that the values of the PPC and Fick are distributed very close to the average with extreme measurements above or below the standard deviation and according to the analysis, the PPC tends to overestimate the value of cardiac output at 3.0L per minute when compared with the reference method (Figure 6) Finally, the utility of the test was calculated by analysis under the ROC curve by the Hanley test, reporting an area of 0.557 (95\% CI 0.464-0.647) (Figure 7).

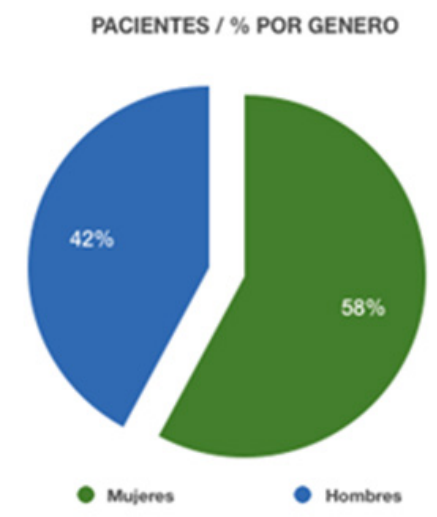

Figure 2 Percentage of patients by gender. 


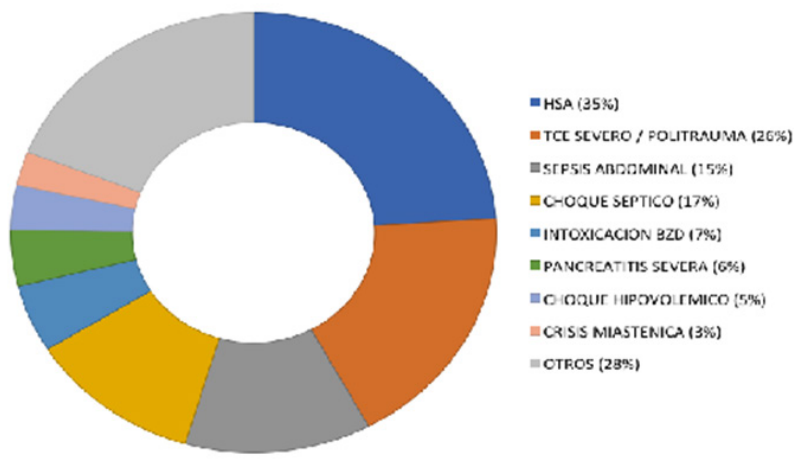

Figure 3 Percentage of incidents of ICU admission pathologies.

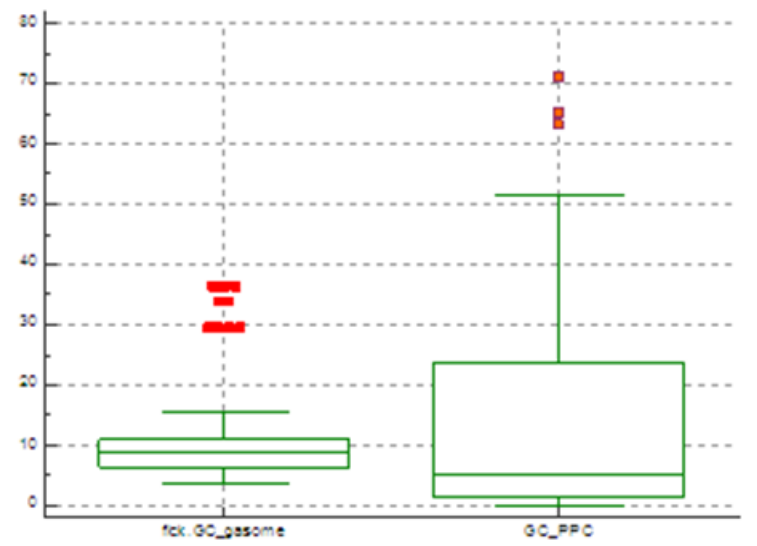

Figure 4 Comparison of the average and standard deviation of the cardiac output obtained by both methods.

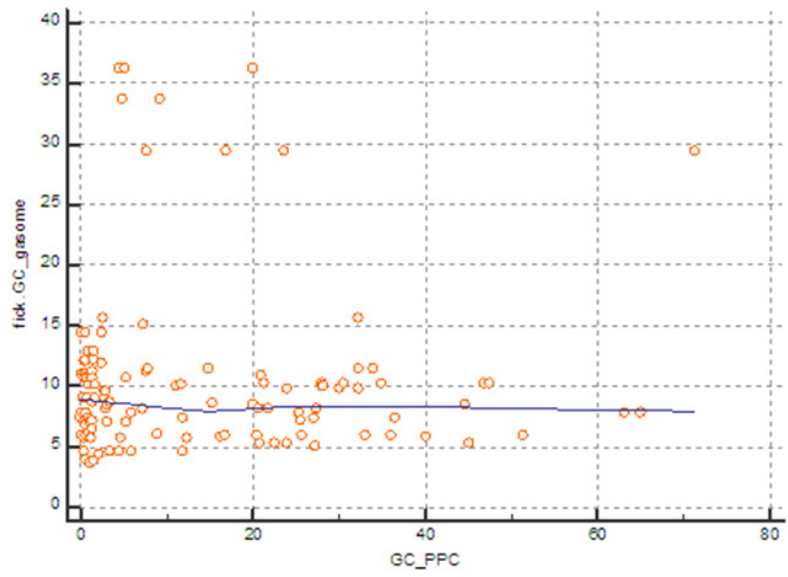

Figure 5 Point dispersion graph demonstrating the correlation of expenditure obtained by PPC with that obtained by the Fick method.

\section{Discussion}

Hemodynamic monitoring is an invaluable tool for the evaluation of critical patients. It allows us not only to detect and determine the origin of hemodynamic instability, but also to guide the choice of the most appropriate treatment and subsequently evaluate its effectiveness. However, monitoring per se is not a therapeutic tool and its use, without a clearly defined objective, does not have to affect the evolution of patients. In order for hemodynamic monitoring to benefit this, it must necessarily be coupled with a treatment protocol that has effectively proven to improve its prognosis. Consequently, the usefulness of monitoring systems should not be assessed only by the accuracy and reliability of their measurements, but also by the ability to favorably affect the evolution of patients. In this sense, many of the arguments used against the use of hemodynamic monitoring have their origin in a non-rationalized use of the same and in the application not directed to specific hemodynamic objectives and of proven benefit for the patient. ${ }^{12}$ The limitations of measurement of cardiac output by transpulmonary thermodilution are: a) thermal variations since there may be thermal artefacts that involve distortion in the thermodilution curve; b) when the patient undergoes extracorporeal purification treatments, the blood is directed to a recirculation path of the thermal indicator and also of artifact; c) intracardiac shunts, due to the distortion on the morphology of the transpulmonary thermodilution curve. ${ }^{13} \mathrm{~A}$ wide variety of methods are currently available to monitor the GC with important differences between them. These devices can be classified according to the degree of invasiveness. According to this classification, we have invasive (CAP), semi-invasive (transpulmonary thermodilution, lithiodilution, pulse wave contour analysis, esophageal Doppler, etc.) and non-invasive (ultrasound, bioreactance, Doppler technology, etc.) systems. ${ }^{14}$

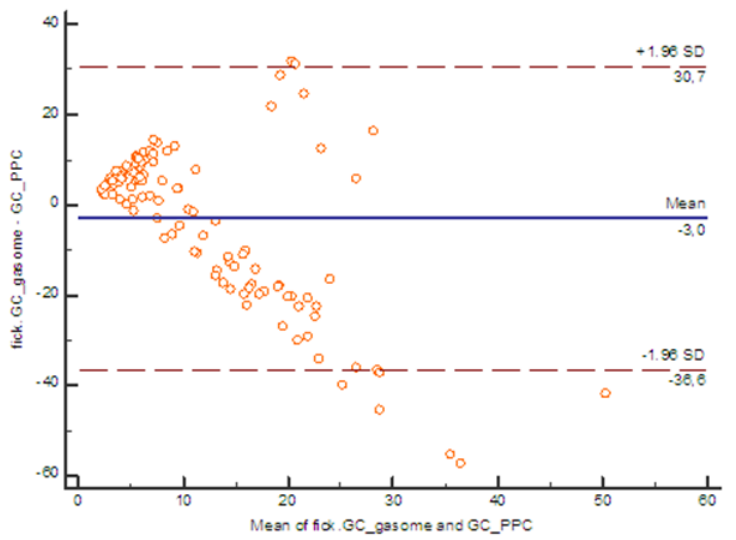

Figure 6 Análisis de Bland-Altman para la concordancia entre ambos métodos de estudio para el gasto cardiaco.

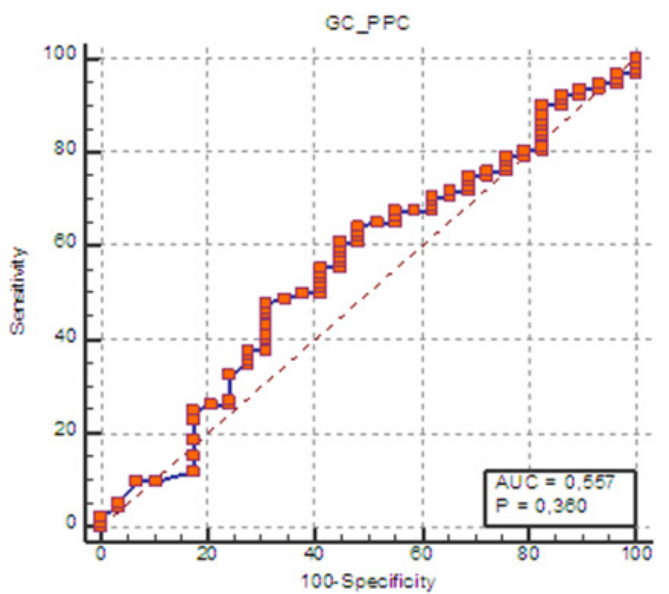

Figure 7 Graph of the area under the ROC curve considering the Fick method as a benchmark or gold standard.

Its main disadvantage is that they lose precision and reliability in situations with significant changes in blood volume and vascular tone change, of particular importance in systems without external calibration and that are still semi-invasive methods with the vascular 
complications that this implies. ${ }^{15}$ Esophageal Doppler estimates blood flow in the descending aorta and calculates vs. The limitations of this technique include operator dependence, which cannot be performed continuously and the poor tolerance of the probe in non-ventilated patients. ${ }^{16,17}$ For several decades, the main method for the determination of GC has been intermittent thermodilution by inserting a catheter into the pulmonary artery (CAP). In recent years, new methods have been developed for the assessment of VS and GC in critically ill patients. However, despite the advances in technology, our unit, like many of our country, lacks invasive devices, so Fick's method, which is our test of reference for project purposes, is easily accessible in addition to its extensive range of reliability in determining cardiac output, with sufficient external validity among experts in the field and is the method that is routinely performed in daily medical practice. ${ }^{18}$

In our study, we compared the average cardiac output with the student $t$ test for independent samples with the measurements obtained by the PPC and with which the Fick method was obtained $(10.42+$ -13.41 vs $10.17+-10.55$ respectively), without finding significant statistical differences $(\mathrm{p}=0.06)$, regarding the objective correlations, the expected relationship between Fick and PPC methods was not found. This may be due to the fact that the Fick method even requires only the obtaining of arterial and venous blood and the determination of $\mathrm{VO} 2$, its estimates are theoretical, based on tables and even arbitrary values.

For a long time, the Fick equation was considered the gold standard for determining cardiac output; but objections are currently described on its realization: the equation was described for "normal" subjects, with hemodynamic stability, a "normal" value of VO2 was established at $250 \mathrm{~mL} / \mathrm{min}$. According to this, Fick's formula assumes important biases for the hemodynamic assessment of critically ill patients, as described in their hemodynamic monitoring study by authors J. Aguirre Sánchez et al. According to this, Fick's method can overestimate cardiac output. up to $20 \%$ compared to the results obtained through the flotation catheter. ${ }^{19}$ As demonstrated in the study conducted by Meyers et al. for monitoring cardiac output in critical patients, performed in Australia, ${ }^{20}$ where they compared hemodynamic monitoring of cardiac output based on transthoracic Doppler with the USCOM- ${ }^{\mathrm{a}}$ monitor, ${ }^{21,22}$ corroborated the advantages of using pulmonary artery catheterization, as a gold standard in the intensive care unit compared to the USCOM-1 monitor, however they report a bias in the study for a small number of patients (mostly post-surgical cardiothoracic patients), and demonstrated that both techniques (cardiac output from the pulmonary artery catheter and the USCOM1st monitor) presented wide limits, especially for overestimating the high cardiac output $(>4 \mathrm{l} / \mathrm{min} / \mathrm{m})$ in which the USCOM did not It is a good GC estimator in correlation to the pulmonary artery catheter, as demonstrated in studies conducted by Van den Oever $^{23}$ \&Thom O et $\mathrm{al}^{24}$ A national study was carried out by Martínez et al, in the areas of intensive therapy and neurointensive therapy of the General Hospital of Ecatepec "Las Américas" in 2017, where they made the comparison of cardiac output measurements by means of echocardiography and USCOM, demonstrating that, with a 95\% confidence interval, the measurement of cardiac output with the USCOM ultrasonic cardiac output monitor and the Doppler echocardiographic evaluation with the application of the continuity equation are comparable measures in patients with critical illness.

\section{Limitations of the study}

We consider that while it is true that we choose an imperfect standard, it is what is used in daily practice. On the other hand, the pulsomagneto cardiac pressure gauge is operator dependent, where the value is underestimated if it is not properly placed on the arterial pulse and if there is tachycardia or tachypnea, it tends to overestimate the value of cardiac output. Similarly, anatomical variants and / or vascular obstructions due to atherosclerosis can lead to measurement bias underestimating the result. It is necessary to carry out new research where other methods of obtaining cardiac output such as echocardiography are compared, to validate the correlation between this and the PPC.

\section{Conclusions}

In our study, the pulsomagneto cardiac pressure gauge is a lowcost device that can be used at the patient's bedside for non-invasive control of cardiac output in critical patients. It has been validated for monitoring the mean arterial pressure; however, it is still in the trial period for the calculation of cardiac output, so new research should be carried out in search of correlation in the recording of cardiac output with other standard methods, such as the echocardiogram. To use monitoring we must have a defined objective in the evolution of patients, so the use of tools for monitoring should result in the accuracy and reliability of the measures in addition to offering a rationalized use according to each patient. The usefulness of a hemodynamic monitoring system should be assessed not only by the ability of the device in question to correctly measure what it has been designed for (such as cardiac output), since most of these systems achieve this goal. In a generally satisfactory way.

\section{Acknowledgments}

The authors thank DAIP-2019/59023 for partial support and the IMSS T1 clinic for their patient availability.

\section{Conflicts of interest}

There are no conflicts of interest.

\section{References}

1. García Delgado M, Rivera Fernández R, De la Chica Ruiz-Ruano R, et al. Análisis de la mortalidad en una unidad de cuidados intensivos neurotraumatologico según el sistema APACHE III. Medicina Intensiva. $2001 ; 25(6)$.

2. Finfer S, Delaney A. Pulmonary artery catheters. BMJ. 2006;333:930931.

3. Trottier SJ, Taylor RW. Physician's attitudes toward and knowledge of the pulmonary catheter. Society of Critical Care Medicine membershio survey. New Horiz. 1997;5:201-206.

4. Parmley CL, Pousman RM. Noinvasive cardiac output monitoring. Curr Opin Anesthesiology. 2002;15(6):675-680.

5. Gil Cano A, Monge Garcia MI, Baigorri González F. Evidencia de la utilidad de la monitorización hemodinámica en el paciente critica. Medicina Intensiva. 2012;36:650-655.

6. Malvivuo J, Plonsey R. Bioelectromagnetism. Principales and applications of bioelectric and biomagnetic fields. New York. Oxford University press; 1995.

7. Kauppinen PK, Hyttinen JAK, Kööbi T, et al. Multiple lead recordings improve accuracy of bio-impedance plethysmographic technique. Medical Engeneering \& Physycus. 1999;21:371-375.

8. Phillips. Datasheet magnetoresistive sensors for magnetic field measurement semiconducors. 1998.

9. Phillips semiconductors magnetoresistive sensors for magnetic field measurement. 2000. 
10. Cano ME, Cordova Fraga T, Sosa M, et al. Understanding the magnetic susceptibility measurements by using an analytical scale. Eur J Phys. 2008;29:345-354.

11. Maldonado Moreles M, Cordova Fraga T, Cano ME, et al. A registration of intravascular pressure curves: magneto-mechanical evaluation. Medical Physics-tenth symposium on medical physics.

12. Baigorri González F, Gil Cano A, Monge García MI. Evidencia de la monitorización hemodinámica en el paciente critico. Medicina intensive. 2012;36:650-655.

13. Carrillo López A, Fiol Sala M, Rodriguez Salgado A. El papel del SwanGanz en la actualidad. Med Intensiva. 2010;34:203-214.

14. Aguirre Sánchez J, Camarena Alejo G, Díaz Esquivel A, et al. Correlación entre el gasto cardiaco por termodilución y medido a través de VCO2. Medicina critica y terapia intensiva. 2012;1:36-41

15. Swan HJC, Ganz W, Forrester JS, et al. Catheterization of the heart in man with the use of a flow-direct ballon tipped catheter. New England J. 1970;283:447-451.

16. Benes J, Chytra I, Altmann P, et al. Intraoperative fluid optimazacion using stroke volumen varation in high surgical patients: results of prospective randomized study. Crit Care. 2010;14:R118.

17. Monge MI, Estella A, Díaz JC, et al. Monitorización hemodinámica minimamente invasiva con eco-doppler esofágico. Medicina intensive. 2008;32:33-44
18. Thom O, Taylor MD, Wolf RE, et al. Comparison of a supra-sternal cardiac output monitor (USCOM) with the pulmonary artery catheter. Br J Anaesth. 2009;103:800-804.

19. 19. Aguirre Sánchez J, Camarena Alejo G, Díaz Esquivel A, et al. Correlación entre el gasto cardiaco por termodilución y medido a través de VCO2. Medicina critica y terapia intensiva. 2012:36-41

20. Cotter G, Williams SG, Vered Z, et al. Role of cardiac power in heart failure. Curr opin cardiology. 2003;18:215-222.

21. Meyer S, Todd D, Wright I, et al. Non-ivasive assessment of cardiac output with portable continuos-wave Doppler ultrasound. Emerg Med Australas. 2008;20:201-208.

22. Van Lelyveld-Hass LE, Van Zanten AR, Borm GF, et al. Clinical validation of the non-invasive cardiac output monitor USCAM- $1^{\mathrm{a}}$ in critically ill patients. Eur J Anaesthesiol. 2008;25:917-924

23. Van den Oever, Murphy EJ, Cristie-Taylor GA. USCOM (ultrasonic cardiac output monitor) lacks agreement with thermodilution cardiaco output and transoesophageal echocardiography valve measurements. Anaesth Intensive Care. 2007;35:903-910.

24. Thom O, Taylor DM, Wolfe RE, et al. Comparison of supra-sternal cardiac output monitor (USCOM) with the pulmonary artery catheter. Br J Anaesth. 2009;103:800-804. 